\title{
Effects of Self-compassion on Diurnal Cortisol Pattern via Positive Affect in Colorectal Cancer Survivors
}

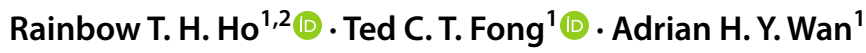

Accepted: 29 October 2021 / Published online: 8 January 2022

(c) The Author(s) 2021

\begin{abstract}
Objectives Colorectal cancer survivors are at risks of emotional distress and dysregulated diurnal cortisol rhythms. Dispositional self-compassion has been linked with better psychological adjustment and greater positive affect. This study evaluated the associations between self-compassion and the diurnal cortisol pattern, and the role of positive affect and emotional distress in mediating this association, in cancer patients.

Methods This longitudinal study recruited 127 Chinese colorectal cancer survivors, who completed assessments for selfcompassion, positive affect, emotional distress, and naturalistic salivary cortisol at baseline. The participants completed follow-up assessments for affect and emotional distress after 2 months (time 2) and the diurnal cortisol pattern after 8 months (time 3). Bootstrapped mediation analysis analyzed the direct and indirect effects of self-compassion on the diurnal cortisol pattern via positive affect and emotional distress.

Results A structural equation model with latent factors of self-compassion, self-criticism, and emotional distress provided an adequate fit to the data. The direct effects of self-compassion and self-criticism on the diurnal cortisol pattern were not significant $(p=0.11-0.50)$. Positive affect, but not emotional distress, at time 2 significantly predicted steeper diurnal cortisol slopes at time $3(\beta=-0.22, \mathrm{SE}=0.08, p<0.01)$. Self-compassion and self-criticism showed significant negative and positive indirect effects on time 3 diurnal cortisol slopes via time 2 positive affect, respectively.

Conclusions Our findings support indirect linkages between self-compassion and steeper diurnal cortisol slopes via positive affect. Positive affect may mediate temporal relationships between self-compassion and neuroendocrine functioning in colorectal cancer survivors.
\end{abstract}

Keywords Emotional distress $\cdot$ Flattened cortisol slopes $\cdot$ Indirect effects $\cdot$ Mediation $\cdot$ Psycho-oncology $\cdot$ Self-criticism

Increased early screening and medical advances have improved the 5-year survival rate for colorectal cancer, which is the second most prevalent cancer in Hong Kong (Zhang et al., 2018). Surgical treatment followed by adjuvant chemotherapy and radiotherapy leave colorectal cancer patients prone to various symptoms such as fatigue (Ho et al., 2014), sleep disturbance (Ho \& Fong, 2014), and

Both Rainbow Ho and Ted Fong share the co-first authorship.

Rainbow T. H. Ho

tinho@hku.hk

1 Centre On Behavioral Health, The University of Hong Kong, The HKJC Building for Interdisciplinary Research, 2/F5 Sassoon Road, Pokfulam, Hong Kong, China

2 Dept of Social Work \& Social Administration, The University of Hong Kong, Pokfulam, Hong Kong, China emotional distress (Fong \& Ho, 2020). Patients are exposed to stressors that disrupt neuroendocrine functioning during the treatment and survivorship stages (Pyter, 2016). The hypothalamic-pituitary-adrenal (HPA) axis is a major neuroendocrine system that controls stress responses via corticosteroids. The HPA axis plays a role in regulating the immune system via downregulation of interleukin-2 production and inhibition of phagocytosis by macrophages. Salivary cortisol is an established stress biomarker that follows a typical diurnal rhythm, showing an initial increase upon awakening that declines gradually during the day (Hellhammer et al., 2009). Dysregulation of the HPA axis, often seen in the form of flattened diurnal cortisol slopes, has been associated with undesirable physical and mental health outcomes (Adam et al., 2017).

Cancer patients are prone to blunted cortisol reactivity (Black et al., 2017) and disruptions of circadian rhythms 
(Sephton \& Spiegel, 2003) which could have adverse implications for cancer prognosis. Flattened diurnal cortisol rhythms have been associated with greater negative affect (Castonguay et al., 2017), anxiety (Armer et al., 2018), perceived stress (Ho et al., 2018), and depression (Sharpley et al., 2017) among cancer patients. Previous longitudinal studies (Cuneo et al., 2017; Garland et al., 2015) indicate a potential role for the diurnal cortisol pattern in psychological adjustment during the survivorship process. Abnormal diurnal circadian cortisol rhythms have been linked with tumor progression (Cash et al., 2015) and decreased survival (Cohen et al., 2012; Schrepf et al., 2015; Sephton et al., 2000 ) in cancer patients. It is clinically important to investigate the behavioral and emotional factors that affect diurnal cortisol rhythms in cancer survivors.

Apart from the conventional psychopathological perspective, recent studies have investigated stress outcomes from a positive psychology perspective. Self-compassion is a holistic construct that refers to being kind and understanding toward oneself when dealing with pain and failure, as opposed to being overly self-critical (Neff, 2003b). It involves the perception of suffering as part of a whole experience rather than as an isolated incident, and approaching painful feelings with mindful awareness rather than overidentifying with them (Neff, 2003a). Dispositional self-compassion has been linked to better psychological adjustment, more effective stress-coping mechanisms (Arambasic et al., 2019), and lower emotional distress in cancer patients (Lennon et al., 2018; Zhu et al., 2019). A recent meta-analysis of 26 studies (Sirois, 2020) found self-compassion to be associated with greater positive affect and lower negative affect and both affect accounted for half of the effects of self-compassion on self-rated health. Positive affect has been linked to steeper diurnal cortisol slopes in healthy adults (Hoyt et al., 2015; Human et al., 2015) and cancer patients (Wang \& Hoyt, 2018).

Positive psychological changes have been theorized to predict enhanced allostasis (Bower et al., 2008), which may buffer the adverse effects of stress and improve physical health. Self-compassion has been associated with lower daily cortisol levels in older adults with various physical health problems (Herriot et al., 2018). From a theoretical perspective, self-compassion may facilitate adaptive psychological responses and ameliorate the biological disturbances that arise as a response to unavoidable stressors. A randomized controlled trial (Kirschner et al., 2019) found that self-compassion was correlated with improvements in positive affect, reduced arousal (lower heart rate), and increased parasympathetic activation (higher heart rate variability). The links between affect and diurnal cortisol pattern in cancer patients (Castonguay et al., 2017; Wang \& Hoyt, 2018) suggest a potential mediating role for affect between self-compassion and diurnal cortisol pattern. A recent study
(Chan et al., 2021) has found moderating effects of selfcompassion against anxiety symptoms but not physiological stress in cancer caregivers.

Given the importance of cortisol in regulation of metabolism and inflammation, it is of practical interests to examine the protective effects of self-compassion on diurnal cortisol pattern in cancer survivors. There were two study objectives for the present study. The first objective was to examine the temporal effects of self-compassion on psychological functioning (positive affect and emotional distress) and diurnal cortisol pattern in colorectal cancer survivors during the survivorship stage. Our second objective was to explore the indirect effects of self-compassion on the diurnal cortisol pattern via positive affect and emotional distress. Such an examination on the physiological effect of self-compassion would contribute to the field in understanding the potential mind-body connection, which could facilitate formulation of clinical interventions for better prognosis of the cancer survivors. This study has several hypotheses: (1) self-compassion has significant effects on positive affect and emotional distress; (2) self-compassion has significant direct effects on the diurnal cortisol pattern; (3) positive affect and emotional distress has significant effects on the diurnal cortisol pattern; and (4) positive affect and emotional distress mediate the relationship between self-compassion and the diurnal cortisol pattern.

\section{Method}

\section{Participants}

The present study comprised a sample of 127 eligible colorectal cancer patients. The majority of the participants were female $(58.3 \%)$ and had received at least 10 years of education $(64.6 \%)$. The mean age of the participants was 63.8 years $(\mathrm{SD}=8.9$, range $=40-85)$ and the average BMI was $22.5(\mathrm{SD}=3.4)$. Most of the participants $(38.7 \%)$ were diagnosed with stage III colorectal cancer, followed by stage I (31.5\%) and stage II (29.7\%). Almost all participants (96\%) had completed surgical treatment. Around half of the sample (44\%) had received chemotherapy and around one-tenth of the sample had received radiotherapy (9.5\%), and complementary and alternative medicine (13.5\%) such as acupuncture, massage, and tai chi that were not part of the standard treatment. The mean number of completed treatments was $1.73(\mathrm{SD}=0.95)$ and the average time elapsed since the last cancer treatment was 1.62 years $(\mathrm{SD}=1.28)$. Less than half of the sample $(41.6 \%)$ reported current use of Western or Chinese medication. Twenty-one participants were lost to follow-up, giving a drop-out rate of $16.5 \%$ for the study over the 8-month study period. The study dropouts $(N=21)$ did not differ significantly from those who completed the study 
$(N=106)$ in terms of demographic characteristics and baseline measures $(p>0.10)$.

Power analysis was conducted using Monte Carlo simulation techniques to determine the sample size requirements for mediation analysis (Wolf et al., 2013). A structural equation model was specified with three observed indicators, with each indicator measuring the predictor, mediator, and outcome variables as latent factors $(\lambda=0.8)$. Standardized path coefficients of 0.14 and 0.39 corresponded to small ( $2 \%$ of the variance) and moderate (13\% of the variance) effect sizes (Miočević et al., 2018). The sample $(N=127)$ showed adequate statistical power (88.3-91.8\%) for detecting indirect effects with moderate magnitudes $(a=b=0.39)$. For indirect effects with small to moderate magnitudes ( $a=0.39$, $b=0.14$ or $a=0.14, b=0.39$ ), the statistical power dropped to $21.0-26.7 \%$.

\section{Procedure}

Study participants were colorectal cancer survivors recruited by convenience sampling through doctor referrals at local hospitals and newsletter advertisements placed at patient resource centers in Hong Kong. Two trained research assistants conducted eligibility screening for 284 patients using the following inclusion criteria: (1) diagnosis of stage I-III colorectal cancer; (2) age 18 years or higher; (3) Cantonesespeaking; (4) expected survival period of $\geq 12$ months; and (5) completion of main cancer treatment (surgery and adjuvant chemotherapy) within 5 years of time of recruitment. A total of 123 patients did not meet the inclusion criteria or were excluded based on the following criteria: (1) presence of severe cachexia, dizziness, or nausea, any of which would prevent moderate physical exertion; (2) diagnosis of medical and mental disorders such as cardiovascular disease, epilepsy, major depressive disorder, generalized anxiety disorder, or schizophrenia; and (3) relapses or metastases of colorectal cancer. Thirty-four patients refused to join the study because of time constraints. Ethical approval was obtained from the Human Research Ethics Committee of the University of Hong Kong (IRB Reference Number: UW 15-115).

This study adopted an 8-month, 3-wave longitudinal study design. At baseline (time 1), the participants provided written informed consent; completed a self-report questionnaire on self-compassion, affect, and emotional distress; and provided salivary cortisol samples. Followup assessments were performed on affect and emotional distress 2 months later (time 2) and salivary cortisol samples for the diurnal cortisol pattern 8 months later (time $3)$. Participants who underwent chemotherapy were prone to various side effects and symptoms throughout the treatment trajectory (Röhrl et al., 2019). Moreover, long-term changes in immune functions (lymphocytes) have been found in cancer survivors 9 months after chemotherapy completion (Verma et al., 2016). Given the likely linkages between immune and endocrine parameters, the present study specified a longer time lag of 6 months between time 2 and time 3 to avoid conflation with the long-term side effects of chemotherapy and investigate the longer-term prognosis of the cancer survivors.

\section{Measures}

Self-compassion The respondents completed the 26-item Self-Compassion Scale (Williams et al., 2014) at time 1. This instrument computes 6 subscale scores as the average of items scored on a 5-point Likert scale $(1=$ never, $5=$ very often). Although the original developer (Neff, 2003a) advocated the use of a total score for self-compassion, recent psychometric findings (Brenner et al., 2017; López et al., 2015) support the use of two aggregate scores for self-compassion and self-criticism. In this study, the model with two secondorder factors provided a significantly better fit $\left(\Delta \chi^{2}=95.8\right.$, $\Delta d f=1, p<0.01$ ) than the model with one second-order factor. This study posited self-compassion as a latent factor measured by the three subscales of self-kindness, common humanity, and mindfulness, and self-criticism as a latent factor measured by the three subscales of self-judgment, isolation, and over-identification. Compared to the widely used Cronbach's alpha $(\alpha)$, McDonald's omega $(\omega)$ is a more general indicator of scale reliability (Hayes \& Coutts, 2020). Satisfactory and good reliability was found for the six subscales $(\omega=0.71-0.82)$ and two aggregate scores of selfcompassion and self-criticism $(\omega=0.91-0.92)$ in the present study, respectively.

Positive Affect and Emotional Distress The participants completed the Chinese version of the 20-item Positive and Negative Affect Schedule (PANAS) and the 14-item Hospital Anxiety and Depression Scale (Fong \& Ho, 2014) at times 1 and 2. The PANAS assesses positive affect and negative affect during the 2 weeks preceding the assessment as the sum of 10 items on a 5 -point Likert scale $(1=$ never, $5=$ very often). The Hospital Anxiety and Depression Scale measures anxiety and depression by summing the 7-item scores using a 4-point format $(0=$ almost never, $3=$ very often $)$. The total scores for positive affect and negative affect range from 10 to 50 and those for anxiety and depression range from 0 to 21 , with higher scores indicating greater distress. In the present study, the continuous score from the PANAS subscale was the observed indicator of positive affect. Emotional distress was posited as a latent variable measured by the continuous scores of anxiety, depression, and negative affect subscales. Good levels of reliability were found for positive affect and negative affect $(\omega=0.88-0.89)$ and anxiety and depressive symptoms $(\omega=0.80-0.86)$ in the present study. 
Diurnal Cortisol Pattern At time 1 and time 3, salivary cortisol samples were collected from the participants using Salivette tubes at 4 different time points during a normal weekday: upon waking, noon (1200 h), late afternoon $(1700 \mathrm{~h})$, and evening (2100 h). The current 1-day, 4-sample cortisol sampling method was adopted in a previous study (Ho et al., 2016). The diurnal cortisol pattern recorded at the 8-month follow-up was regarded as the distal outcome of self-compassion at time 1 and affect at time 2. Participants were instructed to collect the first sample immediately upon waking and to avoid consumption of food and coffee/caffeine drinks (de Haan et al., 2020), smoking, and strenuous exercise for an hour prior to saliva collection at the later time points. Instruction sheets were provided to help the participants collect saliva at the specified times. They were asked to record the collection time of each sample in a daily $\log$. Reminder messages were sent via WhatsApp to improve adherence to the collection protocol. The collected Salivette tubes were maintained in a freezer in the stress laboratory of the university. To determine the cortisol concentrations, the tubes were first thawed and then centrifuged at $3000 \mathrm{rpm}$ for $15 \mathrm{~min}$. An ELISA kit (Salimetrics, USA) was then used to process the samples and calculate the cortisol concentrations. The intra-assay and inter-assay coefficients of variation were $3 \%$ and $10 \%$, respectively.

At time 1, 127 participants provided 508 saliva samples, 485 (95.5\%) of which provided valid cortisol concentrations. At time 3, 424 saliva samples were collected from 106 participants, 401 (94.6\%) of which provided valid cortisol concentrations. After preliminary screening, 9 and 14 outliers ( $>3$ standard deviations [SD] from the mean) were removed from samples obtained at times 1 and 3, respectively. The diurnal cortisol pattern was summarized using the mean cortisol concentration and diurnal cortisol slope. The mean cortisol concentration was derived by dividing the area under the curve with respect to the baseline by the total elapsed time. The diurnal cortisol slope was calculated as the hourly change in cortisol concentration by regressing the four cortisol concentration values on the collection time. Lower (more negative) diurnal slopes indicated a more rapid decline in cortisol levels and slope values closer to zero denoted flatter diurnal rhythms. Previous studies in Chinese samples (Ho et al., 2013; Lai et al., 2005) did not find any significant differences in the diurnal rhythms of cortisol secretion across 2 days of measurement, suggesting acceptable intra-individual stability for the cortisol measures.

\section{Data Analyses}

Structural equation modeling was performed using the robust maximum likelihood estimator in Mplus 8.2 (Muthén \& Muthén, 2017) to estimate the direct and indirect effects of self-compassion and self-criticism on the diurnal cortisol pattern via affect. All of the psychological measures followed a normal distribution with a skewness of $<1$. The latent factors of self-compassion and self-criticism at time 1 were proposed to be predictors of the diurnal cortisol pattern (mean cortisol concentration and diurnal cortisol slope) at time 3 . The observed variables of positive affect and the latent factor of emotional distress at time 2 were hypothesized to be mediator variables. The model included time 1 assessments of affect and the diurnal cortisol pattern to account for autoregressive effects, which describe the stability of individual differences over time. Demographic and clinical characteristics (gender, age, body mass index [BMI], cancer stage, number of treatments completed, time elapsed since last treatment, use of medication) were included as potential confounding variables in the model.

The model fit was assessed using the $\chi^{2}$ test and cutoff criteria for the following approximate fit indices: $p>0.05$ for the $\chi^{2}$ test, comparative fit index (CFI) $\geq 0.95$, and root mean square error of approximation (RMSEA) $\leq 0.06$. To account for the likely skewed distribution, the indirect effects of self-compassion and self-criticism (time 1) on the diurnal cortisol pattern (time 3 ) via affect (time 2) were estimated using 10,000 bootstrap draws. The estimated indirect effects were considered statistically significant if the $95 \%$ confidence interval (CI) excluded zero. Missing data were handled using full information maximum likelihood under the missing-at-random assumption (Little \& Rubin, 2019).

\section{Results}

Table 1 displays the descriptive statistics and bivariate correlations of the study measures from times 1 to 3 . With reference to the theoretical range of the measurement scales, the sample showed moderate levels of self-compassion and positive affect and low levels of self-criticism, negative affect, anxiety, and depression. We found overall stable levels of positive affect (Cohen $d=0.02, p=0.87$ ) and a significant and small decrease in negative affect, anxiety, and depression $(d=0.21-0.25, p=0.01)$ across the 2-month period. The participants showed similar cortisol concentrations (in $\mathrm{nmol} / \mathrm{L})$ of $8.04(\mathrm{SD}=5.48) / 8.74$ $(\mathrm{SD}=5.66)$ in sample $1,3.96(\mathrm{SD}=1.99) / 3.97(\mathrm{SD}=1.89)$ in sample 2, $2.47(\mathrm{SD}=1.31) / 2.40(\mathrm{SD}=1.25)$ in sample 3 , and $1.58(\mathrm{SD}=0.81) / 1.50(\mathrm{SD}=0.77)$ in sample 4 across times 1 and 3 . The sample reported consistent saliva collection time across time 1 and time 3 for all of the 4 samples: sample 1 (mean collection time $=0654 \mathrm{~h}$ and $0656 \mathrm{~h}$ ), sample 2 (1205 h and $1211 \mathrm{~h}$ ), sample $3(1709 \mathrm{~h}$ and $1714 \mathrm{~h})$, and sample $4(2115 \mathrm{~h}$ and $2118 \mathrm{~h})$. The sample displayed overall stability of the diurnal cortisol 


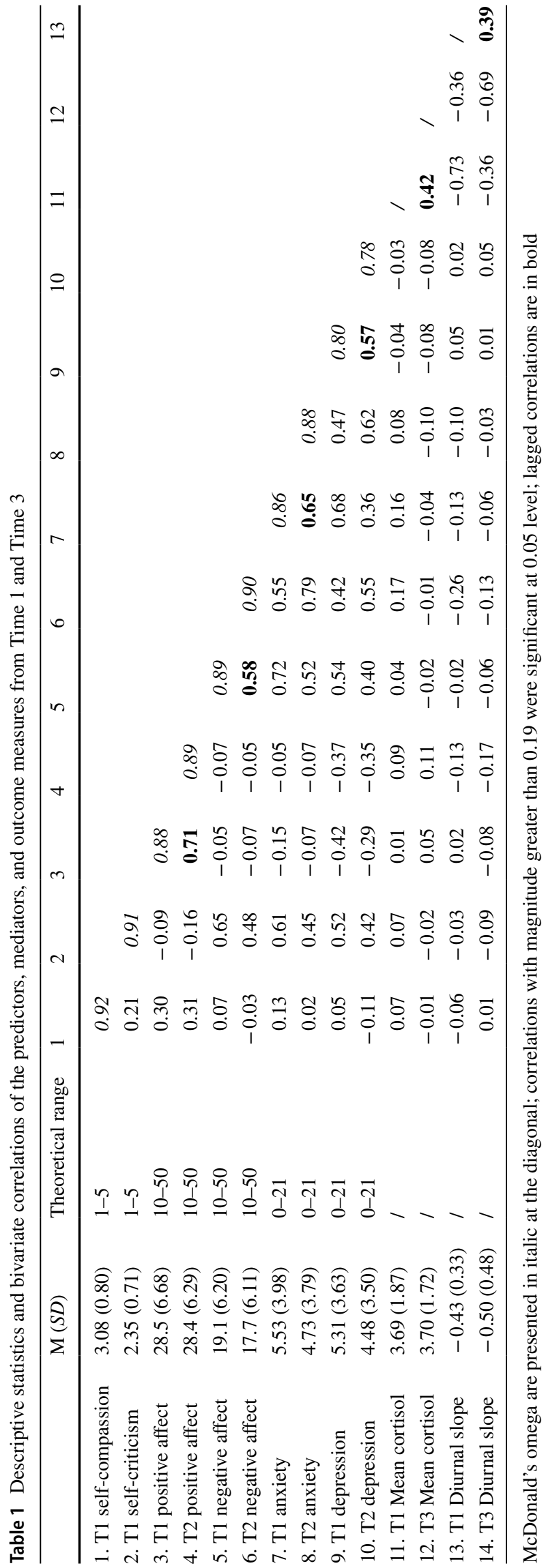

slope and mean cortisol concentration $(d=0.03-0.18$, $p=0.07-0.85$ ) across the 8-month period.

The proposed structural equation model fitted the observed data adequately with $\chi^{2}(132)=159.1, p=0.054$, $\mathrm{CFI}=0.972$, and RMSEA $=0.044$. Figure 1 displays the standardized path estimates of the mediation model. In the figure, the control variables, baseline measures of the stress outcomes, and the direct effects of the time 1 predictors on the time 3 outcomes are omitted for ease of presentation. Gender, BMI, number of treatments completed, time elapsed since last treatment, and use of medication did not show any significant associations $(p>0.05)$ with the study variables in the model. Younger participants and those at more advanced stages of cancer showed greater levels of self-compassion and emotional distress. Substantial factor loading was found for selfcompassion $(\lambda=0.83-0.91)$, self-criticism $(\lambda=0.73-0.82)$, and emotional distress $(\lambda=0.71-0.90)$. We found strong autoregressive effects $(\beta=0.56-0.60$, standard error $[\mathrm{SE}]=0.06-0.13, p<0.01)$ for positive affect and emotional distress from time 1 to time 2 and moderate autoregressive effects $(\beta=0.31-0.33, \mathrm{SE}=0.07-0.09, p<0.01)$ for the diurnal cortisol pattern from time 1 to time 3 .

After controlling for covariates and the baseline affect, self-compassion significantly predicted both positive affect $(\beta=0.20, \mathrm{SE}=0.08, p=0.02)$ and emotional distress $(\beta=-0.19, \mathrm{SE}=0.09, p=0.03)$ at time 2. Self-criticism had a significant negative effect $(\beta=-0.16, \mathrm{SE}=0.08, p=0.04)$ on positive affect at time 2 . The time 2 positive affect had a significant negative effect $(\beta=-0.22, \mathrm{SE}=0.08, p<0.01)$ on the diurnal cortisol slope but not on the mean cortisol concentration $(\beta=0.13, \mathrm{SE}=0.09, p=0.15)$ at time 3 . Time 2 emotional distress did not have any significant effects on the diurnal cortisol pattern $(p=0.19-0.64)$ at time 3 . The model explained over half of the variance in time 2 positive affect and emotional distress $\left(R^{2}=50.3-53.2 \%\right)$. The amount of explained variance in the time 3 mean cortisol concentration and the diurnal cortisol slope was $15.3 \%$ and $16.5 \%$, respectively.

Self-compassion did not have any significant direct effects on the diurnal cortisol slope $(\beta=0.17, \mathrm{SE}=0.11, p=0.11)$ and mean cortisol concentration $(\beta=-0.14, \mathrm{SE}=0.11$, $p=0.19)$. Similarly, the direct effects of self-criticism on the diurnal cortisol slope $(\beta=-0.20, \mathrm{SE}=0.14, p=0.15)$ and mean cortisol concentration $(\beta=0.10, \mathrm{SE}=0.14$, $p=0.50$ ) were not significant. As shown in Table 2, time 1 self-compassion showed a significant and negative indirect effect $(\alpha \beta=-0.043,95 \% \mathrm{CI}=-0.126$ to -0.005$)$ on the time 3 diurnal cortisol slope via time 2 positive affect. There was a significant positive indirect effect $(\alpha \beta=0.036$, $95 \% \mathrm{CI}=0.003-0.108)$ of time 1 self-criticism on the time 3 diurnal cortisol slope via time 2 positive affect. None of the indirect effects via emotional distress on mean cortisol 
Time 1

(Baseline)
Time 1

(Baseline)
Time 2

(2-month follow-up)
Time 3

(8-month follow-up)

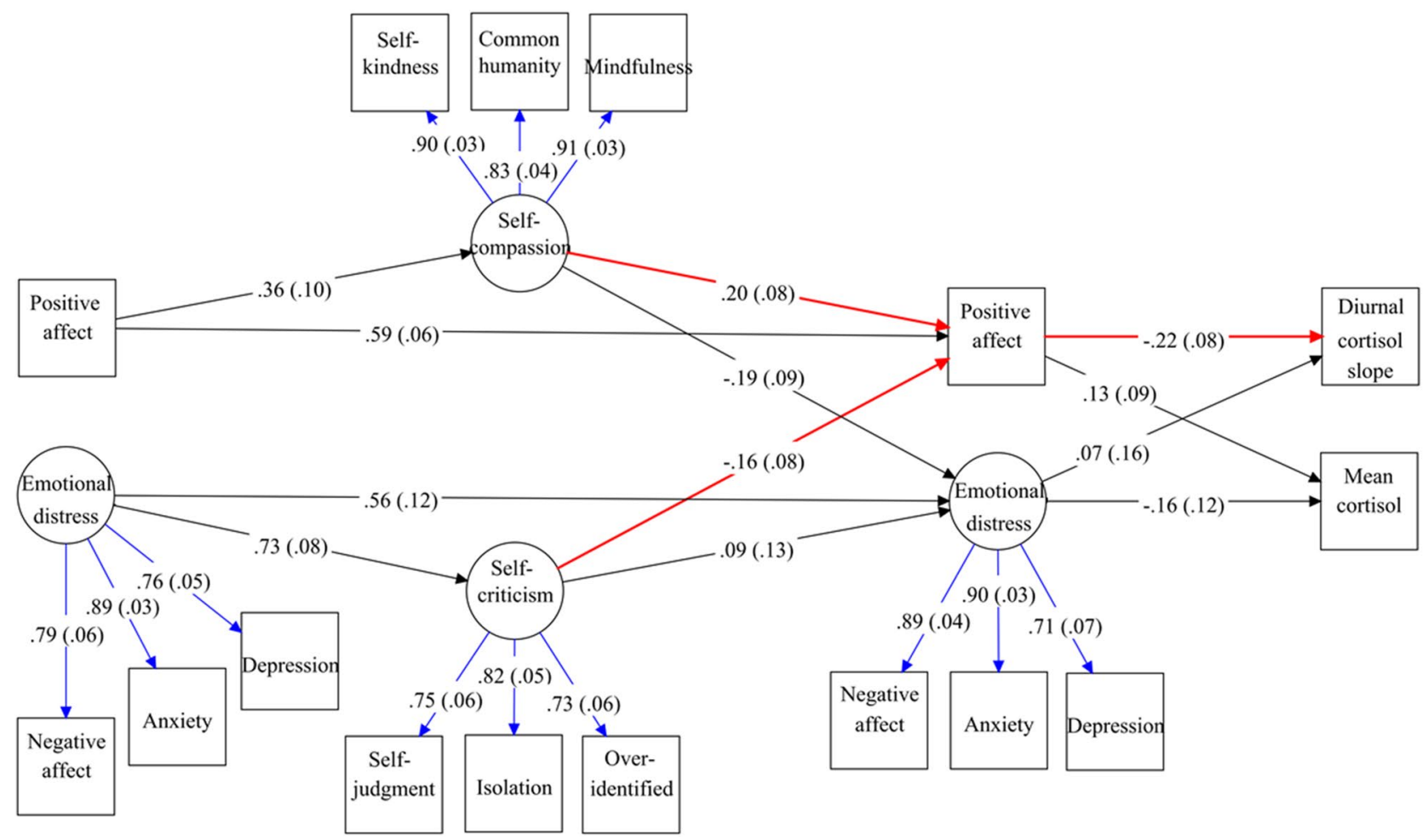

Fig. 1 Standardized path coefficients from self-compassion and selfcriticism at time 1 to the diurnal cortisol pattern at time 3 via positive affect and emotional distress at time 2 . The factor loading of the measurement model is marked in blue and the significant paths of the main study variables are highlighted in bold and red. Standard errors are shown in parentheses. The baseline measures of the stress outcomes, residual co-variances among the dependent variables, and direct effects of the time 1 predictors on the time 3 outcomes are not shown
Table 2 Standardized indirect effects from self-compassion/ self-criticism to stress outcomes via mediating variables

\begin{tabular}{lllcc}
\hline Time 1 predictor & Time 2 mediator & Time 3 outcome & Indirect effect & 95\% confidence interval \\
\hline \multirow{2}{*}{ Self-compassion } & Positive affect & Diurnal cortisol slope & $\mathbf{- 0 . 0 4 3 *}$ & $\mathbf{- 0 . 1 2 6}$ to $\mathbf{- 0 . 0 0 5}$ \\
& Emotional distress & & -0.014 & -0.113 to 0.040 \\
Self-criticism & Positive affect & Diurnal cortisol slope & $\mathbf{0 . 0 3 6}$ & $\mathbf{0 . 0 0 3}$ to 0.108 \\
& Emotional distress & & 0.007 & -0.030 to 0.118 \\
Self-compassion & Positive affect & Mean cortisol & 0.025 & -0.005 to 0.096 \\
& Emotional distress & & 0.030 & -0.009 to 0.129 \\
\multirow{2}{*}{ Self-criticism } & Positive affect & Mean cortisol & -0.021 & -0.087 to 0.004 \\
& Emotional distress & & -0.015 & -0.136 to 0.025 \\
\hline
\end{tabular}

$p<0.05$ with $95 \%$ bootstrapped confidence intervals excluding 0 concentration were statistically significant $(\alpha \beta<0.03)$, with the $95 \%$ bootstrapped CIs including 0 .

\section{Discussion}

This study evaluated the longitudinal associations and contributed to a better understanding between self-compassion and the diurnal cortisol pattern in colorectal cancer survivors. Self-compassion at time 1 significantly predicted greater positive affect and lower emotional distress at time 2 , which lends support to our first hypothesis. The moderate 
correlations found between self-criticism and the components of time 2 emotional distress were consistent with previous studies (Brenner et al., 2017; López et al., 2015). After controlling for baseline emotional distress, self-criticism did not significantly predict time 2 emotional distress. In line with previous findings (Arambasic et al., 2019; Zhu et al., 2019), these results suggest that self-compassion can foster positive affect and ameliorate emotional distress in cancer survivors. Neither self-compassion nor self-criticism had significant direct effects on the diurnal cortisol pattern, which does not support our second hypothesis. The length of time lag ( 8 months) between time 1 and time 3 assessments could undermine the associations between self-compassion and cortisol responses which were acute biomarkers. Indeed, a recent meta-analysis (Phillips \& Hine, 2021) only found a weak correlation $(r=0.14)$ between self-compassion and stress hormones.

This study observed a change in emotional distress but not in positive affect between times 1 and 2. Positive affect at time 2 was found to significantly predict steeper diurnal cortisol slopes 6 months later. The present study used structural equation modeling to model self-compassion, self-criticism, and emotional distress as latent factors and account for the associated measurement errors. Both self-compassion and self-criticism showed significant indirect effects on the diurnal cortisol slope via positive affect but the corresponding indirect effects via emotional distress were not statistically significant. These results provide partial support to the third and fourth hypotheses and suggest that positive affect mediated the link between self-compassion and steeper diurnal cortisol slopes in cancer survivors over an 8-month period. Positive affect has shown a similar mediating role between benefit-finding and diurnal cortisol slopes in prostate cancer patients (Wang \& Hoyt, 2018). Though previous studies (Armer et al., 2018; Castonguay et al., 2017; Pauly et al., 2019) found significant associations between negative affect and the diurnal cortisol pattern, they have not properly accounted for the role of positive affect. Our results lend support to the linkages between diurnal cortisol slopes and positive affect rather than emotional distress (Hoyt et al., 2015; Nater et al., 2010; Wang \& Hoyt, 2018).

One possible explanation for the lack of significant findings between emotional distress measures and the cortisol outcomes could be that the sample was not distressed enough. The mean HADS scores were in the non-clinical range and the relatively low variance in the distress measures could have masked any significance. Future research could test the relationships in a more distressed sample or at a more critical time point in the cancer trajectory. The independent predictive role of positive affect on the diurnal cortisol slope can also be understood from a cultural perspective.
Findings of a recent study (Park et al., 2020) suggest a moderating role for cultural difference, where the association between negative affect and flattened diurnal cortisol slopes was only significant in an American sample but not in a Japanese sample. The discrepancy could reflect divergent cultural beliefs about negative affect being viewed as a threat to self-image in the West, as opposed to being viewed as a natural and integral element of life in the East. The sample of older Chinese individuals in this study likely aligned with Eastern cultural views as they experienced emotions in a dialectic manner (Bagozzi et al., 1999). The stronger emphasis of Chinese culture on feelings of appreciative joy and contentment than Western culture (Zeng et al., 2020) could contribute to a salient role of positive affect in mediating the link between self-compassion and cortisol outcomes.

Our findings on the indirect effects of self-compassion and self-criticism on diurnal cortisol slopes via positive affect integrated multidisciplinary knowledge from positive psychology, neuroendocrinology, and psycho-oncology. Positive affect is a potential pathway by which self-compassion and self-criticism exert their influences on the activities of the HPA axis in cancer survivors. A qualitative study (Goei et al., 2021) reported experiences of increased selfawareness, pleasant body experiences, positive thinking and emotions, and relaxation in adult cancer survivors following mindfulness intervention. Developing self-compassionate attitudes and reducing self-critical attitudes might facilitate positive thinking and overall emotional well-being. Besides, dispositional mindfulness has been found to predict favorable diurnal cortisol pattern in cancer survivors (Garland et al., 2015). Mindfulness-based interventions have shown beneficial effects on the diurnal cortisol pattern in breast cancer survivors (Boyle et al., 2017; Hsiao et al., 2016). Given the potential connections between the endocrine, immune, and metabolic systems (Antoni \& Dhabhar, 2019), a recent pilot randomized controlled trial ( $\mathrm{Ng}$ et al., 2020) on the effectiveness of mindfulness interventions found improvements in the concentrations of C-reactive protein in 55 older adults with mild cognitive impairment. Comparative effectiveness research could highlight the utility of compassion-based and mindfulness-based interventions in modulating physiological stress responses and inform future clinical practice (Conversano et al., 2020).

\section{Limitations and Future Research}

This study is subject to a number of limitations. First, methodological literature (Segerstrom et al., 2014) has suggested the use of multiple $(\geq 3)$ days of saliva assessment to derive reliable estimates for the diurnal cortisol 
pattern. For budgetary reasons, the present study only estimated the mean cortisol and diurnal cortisol slope based on one day of saliva assessment. This casts doubts over the reliability of the cortisol parameters and robustness of the present results. Since diurnal cortisol slopes could alternatively be calculated from only waking and bedtime cortisol as a prognostic marker in cancer samples, further studies could collect fewer cortisol samples per day over more days to obtain more reliable estimates of the diurnal cortisol pattern. Second, though the present study controlled for the general use of medication in the analyses, we did not differentiate between different types of pharmacologic drugs. As a result, we did not specifically measure and control for the use of corticosteroid, which could have potential interference with neuroendocrine functioning in the current results.

Third, the standardized indirect effects obtained in the present study did not reach moderate magnitudes. The small effect sizes imply that this study was likely underpowered when testing these modest indirect effects. Simulation analysis suggests that future studies will require a larger sample size $(N=280)$ to achieve an adequate power of $80 \%$ to analyze modest indirect effects. Fourth, the non-randomized sampling design might result in sampling and self-selection biases. There could be potential recall bias over the past 2 weeks for the questionnaire data collection of the study participants. Our mediation model examined the effects of affect on the diurnal cortisol pattern based on previous literature and did not explore the possible reciprocal effects of diurnal cortisol pattern on affect. We recommend caution in interpreting the temporal ordering of these effects before replication in future longitudinal studies. Fifth, moderator variables were not taken into account given the relatively modest sample size. Future larger-scale studies should consider measuring cancer coping strategies using the MiniMental Adjustment to Cancer Scale (Fong \& Ho, 2015) and test for non-linear effects of self-compassion and self-criticism via moderated mediation analysis.

Based on the present results, future research could test whether self-compassion would be associated with better cancer coping in terms of lower helplessness and anxious preoccupation and higher fighting spirit and explore the potential moderating role of self-compassion on the relationship between cancer coping and psychophysiological functioning in the cancer survivors. Given the prognostic value of cortisol slopes in metastatic populations, future studies should attempt to replicate the present findings in samples of metastatic cancer patients. Randomized clinical trials should be conducted to examine whether compassion-based interventions could foster self-compassion and improve wellbeing of cancer survivors in both the psychological and physiological domains.
Supplementary Information The online version contains supplementary material available at https://doi.org/10.1007/s12671-021-01786-3.

Acknowledgements We would like to express our thanks to Mr. Joshua Yau for his help in the study coordination and cortisol assay and the participants for joining this study.

Author Contribution R. T. H. H.: designed the study, supervised study implementation, acquired funding for the present research, and wrote the paper. T. C. T. F. designed the study, conducted literature review, analyzed the data, interpreted the results, and wrote the paper. A. H. Y. W. collaborated with the study design and editing of the final manuscript.

Funding This work was supported by the General Research Fund of the Hong Kong Research Grants Council (GRF/HKU 17611615).

Data Availability The raw dataset analyzed in this study is available in the form of a supplementary file.

\section{Declarations}

Ethical Approval. The study was conducted in accordance with the ethical standards of the 1964 Helsinki declaration and its later amendments. The study was approved by the ethical review board of the University of Hong Kong (IRB Reference Number: UW 15-115).

Conflict of Interest The authors declare no competing interests.

Informed Consent Informed consent was obtained from all individual participants.

Disclaimer The funder played no role in the collection, analysis, and interpretation of data; in the writing of the report; and in the decision to submit the article for publication.

Open Access This article is licensed under a Creative Commons Attribution 4.0 International License, which permits use, sharing, adaptation, distribution and reproduction in any medium or format, as long as you give appropriate credit to the original author(s) and the source, provide a link to the Creative Commons licence, and indicate if changes were made. The images or other third party material in this article are included in the article's Creative Commons licence, unless indicated otherwise in a credit line to the material. If material is not included in the article's Creative Commons licence and your intended use is not permitted by statutory regulation or exceeds the permitted use, you will need to obtain permission directly from the copyright holder. To view a copy of this licence, visit http://creativecommons.org/licenses/by/4.0/.

\section{References}

Adam, E. K., Quinn, M. E., Tavernier, R., McQuillan, M. T., Dahlke, K. A., \& Gilbert, K. E. (2017). Diurnal cortisol slopes and mental and physical health outcomes: A systematic review and metaanalysis. Psychoneuroendocrinology, 83, 25-41. https://doi.org/ 10.1016/j.psyneuen.2017.05.018

Antoni, M. H., \& Dhabhar, F. S. (2019). The impact of psychosocial stress and stress management on immune responses in patients with cancer. Cancer, 125(9), 1417-1431. https://doi.org/10.1002/ cncr.31943 
Arambasic, J., Sherman, K. A., Elder, E., Network, B. C., \& A. (2019). Attachment styles, self-compassion, and psychological adjustment in long-term breast cancer survivors. Psycho-Oncology, 28(5), 1134-1141. https://doi.org/10.1002/pon.5068

Armer, J. S., Clevenger, L., Davis, L. Z., Cuneo, M., Thaker, P. H., Goodheart, M. J., Bender, D. P., Dahmoush, L., Sood, A. K., Cole, S. W., Slavich, G. M., \& Lutgendorf, S. K. (2018). Life stress as a risk factor for sustained anxiety and cortisol dysregulation during the first year of survivorship in ovarian cancer. Cancer, 124(16), 3401-3408. https://doi.org/10.1002/cncr.31570

Bagozzi, R. P., Wong, N., \& Yi, Y. J. (1999). The role of culture and gender in the relationship between positive and negative affect. Cognition \& Emotion, 13(6), 641-672. https://doi.org/10.1080/ 026999399379023

Black, D. S., Peng, C., Sleight, A. G., Nguyen, N., Lenz, H. J., \& Figueiredo, J. C. (2017). Mindfulness practice reduces cortisol blunting during chemotherapy: A randomized controlled study of colorectal cancer patients. Cancer, 123(16), 3088-3096. https:// doi.org/10.1002/cncr.30698

Bower, J. E., Low, C. A., Moskowitz, J. T., Sepah, S., \& Epel, E. (2008). Benefit finding and physical health: Positive psychological changes and enhanced allostasis. Social and Personality Psychology Compass, 2(1), 223-244. https://doi.org/10.1111/j.1751-9004. 2007.00038.x

Boyle, C. C., Stanton, A. L., Ganz, P. A., Crespi, C. M., \& Bower, J. E. (2017). Improvements in emotion regulation following mindfulness meditation: Effects on depressive symptoms and perceived stress in younger breast cancer survivors. Journal of Consulting and Clinical Psychology, 85(4), 397-402. https:// doi.org/10.1037/ccp0000186

Brenner, R. E., Heath, P. J., Vogel, D. L., \& Credé, M. (2017). Two is more valid than one: Examining the factor structure of the SelfCompassion Scale (SCS). Journal of Counseling Psychology, 64(6), 696-707. https://doi.org/10.1037/cou0000211

Cash, E., Sephton, S. E., Chagpar, A. B., Spiegel, D., Rebholz, W. N., Zimmaro, L. A., Tillie, J. M., \& Dhabhar, F. S. (2015). Circadian disruption and biomarkers of tumor progression in breast cancer patients awaiting surgery. Brain, Behavior, and Immunity, 48, 102-114. https://doi.org/10.1016/j.bbi.2015.02.017

Castonguay, A. L., Wrosch, C., \& Sabiston, C. M. (2017). The roles of negative affect and goal adjustment capacities in breast cancer survivors: Associations with physical activity and diurnal cortisol secretion. Health Psychology, 36(4), 320-331. https:// doi.org/10.1037/hea0000477

Chan, E. W. W., Liang, L., Liu, N. H., \& Hou, W. K. (2021). The moderated associations of self-compassion with physiological and psychological stress responses: Comparisons between cancer caregivers and non-caregivers. Journal of Health Psychology. https://doi.org/10.1177/13591053211030994

Cohen, L., Cole, S. W., Sood, A. K., Prinsloo, S., Kirschbaum, C., Arevalo, J. M. G., Jennings, N. B., Scott, S., Vence, L., Wei, Q., Kentor, D., Radvanyi, L., Tannir, N., Jonasch, E., Tamboli, P., \& Pisters, L. (2012). Depressive symptoms and cortisol rhythmicity predict survival in patients with renal cell carcinoma: Role of inflammatory signaling. PLoS ONE, 7(8), e42324. https://doi. org/10.1371/journal.pone.0042324

Conversano, C., Ciacchini, R., Orrù, G., Di Giuseppe, M., Gemignani, A., \& Poli, A. (2020). Mindfulness, compassion, and self-compassion among health care professionals: What's new? A systematic review. Frontiers in Psychology, 11, 1683. https:// doi.org/10.3389/fpsyg.2020.01683

Cuneo, M. G., Schrepf, A., Slavich, G. M., Thaker, P. H., Goodheart, M., Bender, D., Cole, S. W., Sood, A. K., \& Lutgendorf, S. K. (2017). Diurnal cortisol rhythms, fatigue and psychosocial factors in five-year survivors of ovarian cancer.
Psychoneuroendocrinology, 84, 139-142. https://doi.org/10. 1016/j.psyneuen.2017.06.019

de Haan, R. E., Blankert, M., \& MohammadiZiabari, S. S. (2020). Integrative biological, cognitive and affective modeling of caffeine use on stress. In F. Herrera, K. Matsui, \& S. Rodríguez-González (Eds.), Distributed Computing and Artificial Intelligence, 16th International Conference.

Fong, T. C. T., \& Ho, R. T. H. (2014). Testing gender invariance of the Hospital Anxiety and Depression Scale using the classical approach and Bayesian approach. Quality of Life Research, 23(5), 1421-1426. https://doi.org/10.1007/s11136-013-0594-3

Fong, T. C. T., \& Ho, R. T. H. (2015). Re-examining the factor structure and psychometric properties of the Mini-Mental Adjustment to Cancer Scale in a sample of 364 Chinese cancer patients. Supportive Care in Cancer, 23(2), 353-358. https://doi.org/10.1007/ s00520-014-2367-0

Fong, T. C. T., \& Ho, R. T. H. (2020). Mindfulness facets predict quality of life and sleep disturbance via physical and emotional distresses in Chinese cancer patients: A moderated mediation analysis. Psycho-Oncology, 29(5), 894-901. https://doi.org/10. 1002/pon.5363

Garland, E. L., Beck, A. C., Lipschitz, D. L., \& Nakamura, Y. (2015). Dispositional mindfulness predicts attenuated waking salivary cortisol levels in cancer survivors: A latent growth curve analysis. Journal of Cancer Survivorship, 9(2), 215-222. https://doi.org/ 10.1007/s11764-014-0402-2

Goei, L. P. S., Lopez, V., \& Klainin-Yobas, P. (2021). Exploring the perceptions of cancer survivors of a mindfulness intervention at a tertiary hospital in Singapore: A descriptive qualitative study. Supportive Care in Cancer, 29, 2723-2733. https://doi.org/10. 1007/s00520-020-05792-4

Hayes, A. F., \& Coutts, J. J. (2020). Use omega rather than Cronbach's alpha for estimating reliability. But... Communication Methods and Measures, 14(1), 1-24. https://doi.org/10.1080/19312458. 2020.1718629

Hellhammer, D. H., Wüst, S., \& Kudielka, B. M. (2009). Salivary cortisol as a biomarker in stress research. Psychoneuroendocrinology, 34(2), 163-171. https://doi.org/10.1016/j.psyneuen.2008.10.026

Herriot, H., Wrosch, C., \& Gouin, J.-P. (2018). Self-compassion, chronic age-related stressors, and diurnal cortisol secretion in older adulthood. Journal of Behavioral Medicine, 41(6), 850-862. https://doi.org/10.1007/s10865-018-9943-6

Ho, R. T. H., \& Fong, T. C. T. (2014). Factor structure of the Chinese version of the Pittsburgh Sleep Quality Index in breast cancer patients. Sleep Medicine, 15(5), 565-569. https://doi.org/10. 1016/j.sleep.2013.10.019

Ho, R. T. H., Fong, T. C. T., Chan, C. K. P., \& Chan, C. L. W. (2013). The associations between diurnal cortisol patterns, self-perceived social support, and sleep behavior in Chinese breast cancer patients. Psychoneuroendocrinology, 38(10), 2337-2342. https:// doi.org/10.1016/j.psyneuen.2013.05.004

Ho, R. T. H., Fong, T. C. T., \& Cheung, I. K. M. (2014). Cancer-related fatigue in breast cancer patients: Factor mixture models with continuous non-normal distributions. Quality of Life Research, 23(10), 2909-2916. https://doi.org/10.1007/s11136-014-0731-7

Ho, R. T. H., Fong, T. C. T., Wan, A. H. Y., Au-Yeung, F. S. W., Wong, C. P. K., Ng, W. Y. H., Cheung, I. K. M., Lo, P. H. Y., Ng, S. M., \& Chan, C. L. W. (2016). A randomized controlled trial on the psychophysiological effects of physical exercise and Tai-chi in patients with chronic schizophrenia. Schizophrenia Research, 171(1), 42-49. https://doi.org/10.1016/j.schres.2016.01.038

Ho, R. T. H., Fong, T. C. T., \& Yip, P. S. F. (2018). Perceived stress moderates the effects of a randomized trial of dance movement therapy on diurnal cortisol slopes in breast cancer patients. Psychoneuroendocrinology, 87, 119-126. https://doi.org/10.1016/j. psyneuen.2017.10.012 
Hoyt, L. T., Craske, M. G., Mineka, S., \& Adam, E. K. (2015). Positive and negative affect and arousal: Cross-sectional and longitudinal associations with adolescent cortisol diurnal rhythms. Psychosomatic Medicine, 77(4), 392-401. https://doi.org/10.1097/psy. 0000000000000178

Hsiao, F.-H., Jow, G.-M., Kuo, W.-H., Yang, P.-S., Lam, H.-B., Chang, K.-J., Lee, J.-J., Huang, C.-S., Lai, Y.-M., \& Chen, Y.-T. (2016). The long-term effects of mindfulness added to family resilienceoriented couples support group on psychological well-being and cortisol responses in breast cancer survivors and their partners. Mindfulness, 7(6), 1365-1376. https://doi.org/10.1007/ s12671-016-0578-9

Human, L. J., Whillans, A. V., Hoppmann, C. A., Klumb, P., Dickerson, S. S., \& Dunn, E. W. (2015). Finding the middle ground: Curvilinear associations between positive affect variability and daily cortisol profiles. Emotion, 15(6), 705-720. https://doi.org/ 10.1037/emo0000071

Kirschner, H., Kuyken, W., Wright, K., Roberts, H., Brejcha, C., \& Karl, A. (2019). Soothing your heart and feeling connected: A new experimental paradigm to study the benefits of self-compassion. Clinical Psychological Science, 7(3), 545-565. https://doi. org/10.1177/2167702618812438

Lai, J. C. L., Evans, P. D., Ng, S. H., Chong, A. M. L., Siu, O. T., Chan, C. L. W., Ho, S. M. Y., Ho, R. T. H., Chan, P., \& Chan, C. C. (2005). Optimism, positive affectivity, and salivary cortisol. British Journal of Health Psychology, 10(4), 467-484. https:// doi.org/10.1348/135910705x26083

Lennon, J., Hevey, D., \& Kinsella, L. (2018). Gender role conflict, emotional approach coping, self-compassion, and distress in prostate cancer patients: A model of direct and moderating effects. Psycho-Oncology, 27(8), 2009-2015. https://doi.org/10.1002/ pon. 4762

Little, R. J. A., \& Rubin, D. B. (2019). Statistical analysis with missing data (3rd ed., Vol. 793). John Wiley \& Sons.

López, A., Sanderman, R., Smink, A., Zhang, Y., van Sonderen, E., Ranchor, A., \& Schroevers, M. J. (2015). A reconsideration of the Self-Compassion Scale's total score: Self-compassion versus selfcriticism. PLOS ONE, 10(7), e0132940. https://doi.org/10.1371/ journal.pone.0132940

Miočević, M., O'Rourke, H. P., MacKinnon, D. P., \& Brown, H. C. (2018). Statistical properties of four effect-size measures for mediation models. Behavior Research Methods, 50(1), 285-301. https://doi.org/10.3758/s13428-017-0870-1

Muthén, L. K., \& Muthén, B. O. (2017). Mplus user's guide (8th ed.). Muthén \& Muthén.

Nater, U. M., Hoppmann, C., \& Klumb, P. L. (2010). Neuroticism and conscientiousness are associated with cortisol diurnal profiles in adults-Role of positive and negative affect. Psychoneuroendocrinology, 35(10), 1573-1577. https://doi.org/10.1016/j.psyneuen. 2010.02.017

Neff, K. D. (2003a). The development and validation of a scale to measure self-compassion. Self and Identity, 2(3), 223-250. https:// doi.org/10.1080/15298860309027

Neff, K. D. (2003b). Self-compassion: An alternative conceptualization of a healthy attitude toward oneself. Self and Identity, 2(2), 85-101. https://doi.org/10.1080/15298860309032

Ng, T. K. S., Fam, J., Feng, L., Cheah, I.K.-M., Tan, C.T.-Y., Nur, F., Wee, S. T., Goh, L. G., Chow, W. L., Ho, R.C.-M., Kua, E. H., Larbi, A., \& Mahendran, R. (2020). Mindfulness improves inflammatory biomarker levels in older adults with mild cognitive impairment: A randomized controlled trial. Translational Psychiatry, 10(1), 21. https://doi.org/10.1038/s41398-020-0696-y

Park, J., Kitayama, S., Miyamoto, Y., \& Coe, C. L. (2020). Feeling bad is not always unhealthy: Culture moderates the link between negative affect and diurnal cortisol profiles. Emotion, 20(5), $721-$ 733. https://doi.org/10.1037/emo0000605

Pauly, T., Michalowski, V. I., Natcr, U. M., Gerstorf, D., Ashe, M. C., Madden, K. M., \& Hoppmann, C. A. (2019). Everyday associations between older adults' physical activity, negative affect, and cortisol. Health Psychology, 38(6), 494-501. https://doi.org/10. 1037/hea0000743

Phillips, W. J., \& Hine, D. W. (2021). Self-compassion, physical health, and health behaviour: A meta-analysis. Health Psychology Review, 15(1), 113-139. https://doi.org/10.1080/17437199. 2019.1705872

Pyter, L. M. (2016). The influence of cancer on endocrine, immune, and behavioral stress responses. Physiology \& Behavior, 166, 4-13. https://doi.org/10.1016/j.physbeh.2015.09.031

Röhrl, K., Guren, M. G., Småstuen, M. C., \& Rustøen, T. (2019). Symptoms during chemotherapy in colorectal cancer patients. Supportive Care in Cancer, 27(8), 3007-3017. https://doi.org/ 10.1007/s00520-018-4598-y

Schrepf, A., Thaker, P. H., Goodheart, M. J., Bender, D., Slavich, G. M., Dahmoush, L., Penedo, F., DeGeest, K., Mendez, L., Lubaroff, D. M., Cole, S. W., Sood, A. K., \& Lutgendorf, S. K. (2015). Diurnal cortisol and survival in epithelial ovarian cancer. Psychoneuroendocrinology, 53, 256-267. https://doi.org/10. 1016/j.psyneuen.2015.01.010

Segerstrom, S. C., Boggero, I. A., Smith, G. T., \& Sephton, S. E. (2014). Variability and reliability of diurnal cortisol in younger and older adults: Implications for design decisions. Psychoneuroendocrinology, 49, 299-309. https://doi.org/10.1016/j.psyne uen.2014.07.022

Sephton, S., \& Spiegel, D. (2003). Circadian disruption in cancer: A neuroendocrine-immune pathway from stress to disease? Brain, Behavior, and Immunity, 17(5), 321-328. https://doi.org/10.1016/ S0889-1591(03)00078-3

Sephton, S. E., Sapolsky, R. M., Kraemer, H. C., \& Spiegel, D. (2000). Diurnal cortisol rhythm as a predictor of breast cancer survival. Journal of the National Cancer Institute, 92(12), 994-1000. https://doi.org/10.1093/jnci/92.12.994

Sharpley, C. F., Christie, D. R. H., Bitsika, V., Agnew, L. L., Andronicos, N. M., McMillan, M. E., \& Richards, T. M. (2017). The use of salivary cortisol as an index of chronic stress that correlates with depression in prostate cancer patients. Psycho-Oncology, 26(9), 1400-1402. https://doi.org/10.1002/pon.4327

Sirois, F. M. (2020). The association between self-compassion and self-rated health in 26 samples. BMC Public Health, 20(1), 74. https://doi.org/10.1186/s12889-020-8183-1

Verma, R., Foster, R. E., Horgan, K., Mounsey, K., Nixon, H., Smalle, N., Hughes, T. A., \& Carter, C. R. D. (2016). Lymphocyte depletion and repopulation after chemotherapy for primary breast cancer. Breast Cancer Research, 18(1), 10. https://doi.org/10.1186/ s13058-015-0669-x

Wang, A. W., \& Hoyt, M. A. (2018). Benefit finding and diurnal cortisol after prostate cancer: The mediating role of positive affect. Psycho-Oncology, 27(4), 1200-1205. https://doi.org/10.1002/ pon. 4647

Williams, M. J., Dalgleish, T., Karl, A., \& Kuyken, W. (2014). Examining the factor structures of the five facet mindfulness questionnaire and the self-compassion scale. Psychological Assessment, 26(2), 407-418. https://doi.org/10.1037/a0035566

Wolf, E. J., Harrington, K. M., Clark, S. L., \& Miller, M. W. (2013). Sample size requirements for structural equation models: An evaluation of power, bias, and solution propriety. Educational and Psychological Measurement, 76(6), 913-934. https://doi.org/10. 1177/0013164413495237 
Zeng, X., Sun, Y., Deng, Y., \& Oei, T. P. S. (2020). Appreciative joy rooted in Chinese culture: Its relationship with strengths in values in action. The Journal of Positive Psychology, 15(1), 112-121. https://doi.org/10.1080/17439760.2019.1651886

Zhang, B., Xie, S.-H., \& Yu, I.T.-S. (2018). Differential incidence trends of colon and rectal cancers in Hong Kong: An age-periodcohort analysis. Cancer Communications, 38(1), 42. https://doi. org/10.1186/s40880-018-0311-2
Zhu, L., Yao, J. T., Wang, J., Wu, L. Y., Gao, Y. H., Xie, J., Liu, A. L., Ranchor, A. V., \& Schroevers, M. J. (2019). The predictive role of self-compassion in cancer patients' symptoms of depression, anxiety, and fatigue: A longitudinal study. Psycho-Oncology, 28(9), 1918-1925. https://doi.org/10.1002/pon.5174

Publisher's Note Springer Nature remains neutral with regard to jurisdictional claims in published maps and institutional affiliations. 